\title{
Development and deployment of a rapid recombinase polymerase amplification Ebola virus detection assay in Guinea in 2015
}

O Faye ${ }^{12}$, O Faye ${ }^{12}$, B Soropogui ${ }^{3}$, P Patel ${ }^{4}$, AA El Wahed ${ }^{5}$, C Loucoubar ${ }^{6}$, G Fall ${ }^{1}$, D Kiory ${ }^{1}$, N Magassouba ${ }^{3}$, S Keita $^{7}$, MK

Kondé ${ }^{8}$, AA Diallo ${ }^{7}$, L Koivogui 9 , H Karlberg ${ }^{10}$, A Mirazimi ${ }^{10}{ }^{11}, O$ Nentwich ${ }^{12}, O$ Piepenburg ${ }^{12}, M^{~ N i e d r i g ~}{ }^{4}, M$ Weidmann $^{213}{ }^{14}$, AA Sall 1214

1. Arbovirus and viral hemorragic fever unit, Institut Pasteur de Dakar, Dakar, Senegal

2. These authors contributed equally to the paper

3. Laboratory for Hemorrhagic fevers of Guinea, Conakry, Guinea

4. Centre for Biological Threats and Special Pathogens, Robert Koch Institute, Berlin, Germany

5. Unit of Infection Models, German Primate Center, Goettingen, Germany, currently Division of Microbiology and Animal Hygiene, Georg-August-University, Goettingen, Germany

6. Group of Biostatistics, Bioinformatics and Modeling, Institut Pasteur de Dakar, Dakar, Senegal

7. Ministry of Health, Conakry, Guinea

8. Center for Research and training on malaria and prioritary diseases (CEFORPAG), Conakry, Guinea

9. National Public Health Institute, Conakry, Guinea

10. Public Health Agency of Sweden, Solna Sweden,

11. Karolinska Institute, Solna, Sweden

12. TwistDx, Babraham Research Campus, Babraham, Cambridge, United Kingdom

13. Institute of Aquaculture, University of Stirling, Stirling, United Kingdom

14. These authors jointly supervised the project

Correspondence: Manfred Weidmann (m.w.weidmann@stir.ac.uk)

Citation style for this article:

Faye O, Faye O, Soropogui Bé, Patel P, El Wahed AA, Loucoubar C, Fall G, Kiory D, Magassouba N'F, Keita S, Kondé MK, Diallo AA, Koivogui L, Karlberg H, Mirazimi A, Nentwich O, Piepenburg 0, Niedrig M, Weidmann M, Sall AA. Development and deployment of a rapid recombinase polymerase amplification Ebola virus detection assay in Guinea in 2015. Euro Surveill. 2015;20(44):pii=30053. DOI: http://dx.doi.org/10.2807/1560-7917.ES.2015.20.44.30053

Article submitted on 27 July 2015 / accepted on 28 September 2015 / published on 05 November 2015

In the absence of a vaccine or specific treatments for Ebola virus disease (EVD), early identification of cases is crucial for the control of EVD epidemics. We evaluated a new extraction kit (SpeedXtract (SE), Qiagen) on sera and swabs in combination with an improved diagnostic reverse transcription recombinase polymerase amplification assay for the detection of Ebola virus (EBOV-RT-RPA). The performance of combined extraction and detection was best for swabs. Sensitivity and specificity of the combined SE and EBOV-RT-RPA were tested in a mobile laboratory consisting of a mobile glovebox and a Diagnostics-in-a-Suitcase powered by a battery and solar panel, deployed to Matoto Conakry, Guinea as part of the reinforced surveillance strategy in April 2015 to reach the goal of zero cases. The EBOV-RT-RPA was evaluated in comparison to two real-time PCR assays. Of 928 post-mortem swabs, 120 tested positive, and the combined SE and EBOV-RTRPA yielded a sensitivity and specificity of $100 \%$ in reference to one real-time RT-PCR assay. Another widely used real-time RT-PCR was much less sensitive than expected. Results were provided very fast within 30 to $60 \mathrm{~min}$, and the field deployment of the mobile laboratory helped improve burial management and community engagement.

\section{Introduction}

As of 11 October 2015, the ongoing Ebola virus disease (EVD) epidemic in West Africa has resulted in more than 28,500 cases and over 11,300 deaths. The early symptoms of EVD (i.e. fever, fatigue, headache, vomiting and diarrhoea) are unspecific and present a challenge for clinical diagnosis [1]. In humans, death occurs generally seven to 10 days after the onset of symptoms. Survivors can be ill for up to 22 days before recovering [2]. Ebola virus (EBOV) infection is mainly diagnosed by various in-house and commercial real-time RT-PCR assays [3], used in up to 38 laboratories implemented at or close to Ebola treatment centres (ETC) in West Africa [4]. Transmission of EVD occurs almost exclusively from human to human by direct contact with body fluids of symptomatic cases. Consequently, the control strategy for EVD epidemics relies on early identification of EBOV-infected patients and corpses for, respectively, isolation and safe burials. It is imperative to trace and follow up contacts and to implement infection control measures.

Therefore, rapid EVD diagnostics impact on outcome of treatment, efficiency of contact tracing and subsequently community engagement, which is central to the successful control of the EVD epidemic. The World Health Organization (WHO) launched a call and consultation for an emergency procedure under its 


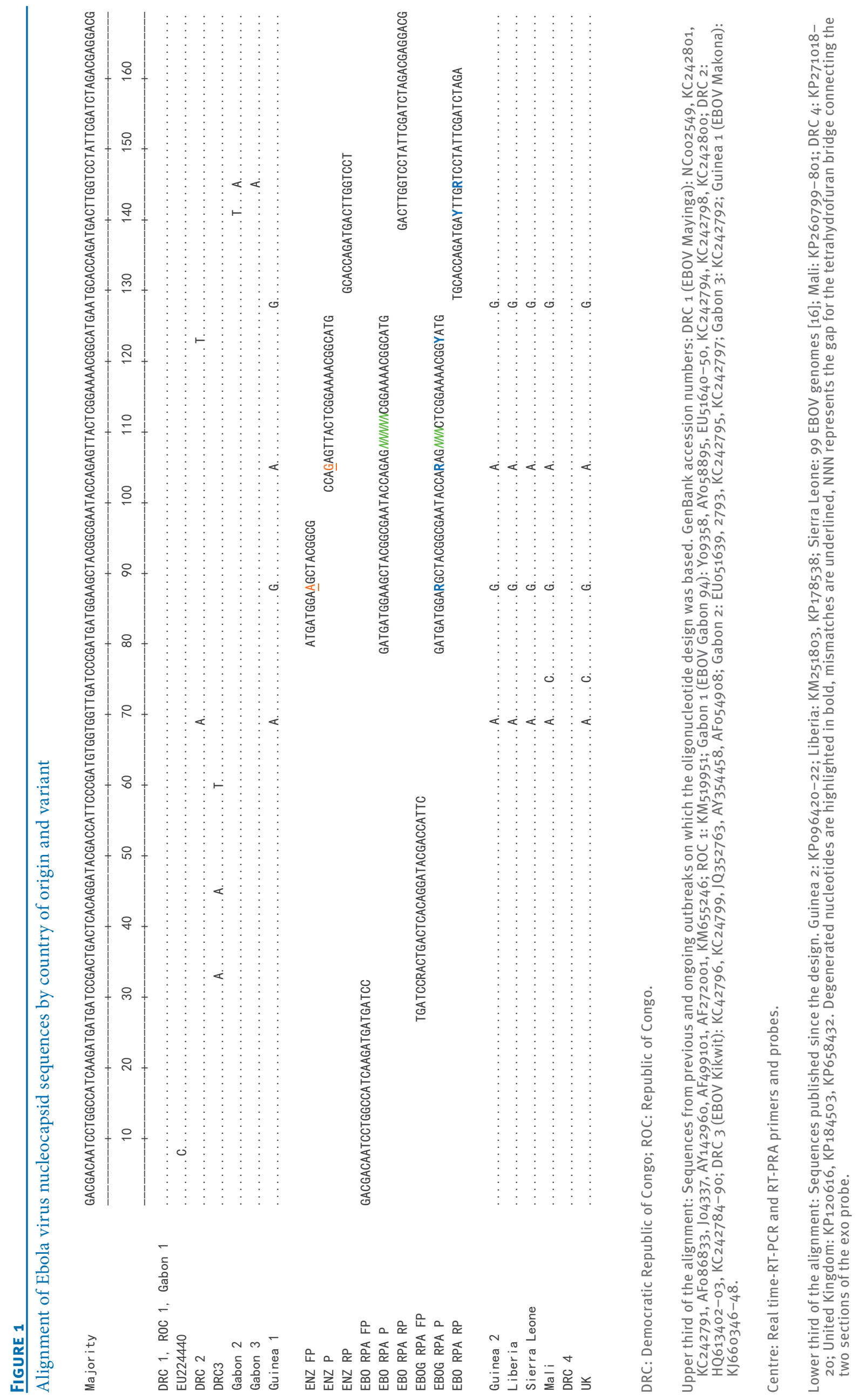




\section{FIGURE 2}

Mobile laboratory for Ebola virus diagnostics
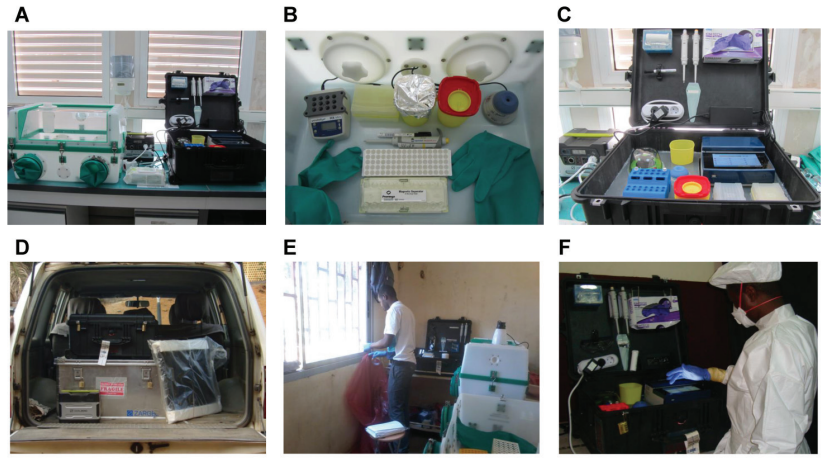

E

$\mathbf{F}$
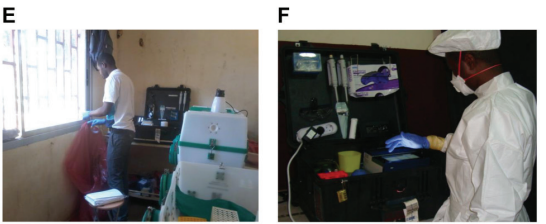

A: Complete mobile laboratory including the solar power pack.

B: The Diagnostics-in-a-Suitcase (DiaS) contains all equipment and reagents to perform up to 100 RT-RPA assays

C: The workspace inside the glovebox contained a heat block mini (VWR International GmbH, Erlangen, Germany), a 96 box each of $100 \mu \mathrm{l}$ and $1,000 \mu \mathrm{l}$ sterile filter tips (BRAND, Wertheim, Germany), and two respective automatic micropipettes (Eppendorf AG, Hamburg, Germany), a waste container (Sarstedt, Nuembrecht, Germany), a magnetic separator stand (Promega, Madison, US), a rack for 1.5-2 $\mathrm{ml}$ tubes and a marker pen.

D: Transfer of the mobile laboratory (DiaS, aluminium box containing the glovebox and the PPE, solar panel and power pack).

E: Setup of the glovebox and the DiaS at a hospital in Matoto.

F: Ebola RT-RPA assay in the DiaS.

pre-qualification programme for diagnostic tool assessment [5] to support accelerated development, production and deployment of adapted and rapid Ebola tests. Early in 2015, only three commercial real-time RT-PCR assays (RealStar Filovirus Screen, Altona Diagnostics, Hamburg, Germany; Liferiver Ebola Virus, Shanghai ZJ BioTech Co., Shanghai, China; GeneXpert Ebola virus, Cepheid, Solna, Sweden) and one rapid antigen detection test (ReEBOVTM (Corgenix, Denver, United States (US)) had been approved for emergency use, emphasising the need for such tests. At the time of publication of this article, nine real-time PCR assays for Ebola virus detection have been approved by the WHO.

In this study, we describe the optimisation, evaluation of performance and operational characteristics of a real-time RT-PCR [6] and a rapid RT-recombinase polymerase amplification (RPA) [7] used for diagnosis of suspected Ebola cases, and compare them with the RealStar Filovirus Screen RT-PCR approved for emergency use. In addition, we report the efficient field deployment of the rapid RT-RPA which boosted community engagement for safe and dignified burials.
FIGURE 3

Assembly of the Diagnostics-in-a-Suitcase
A

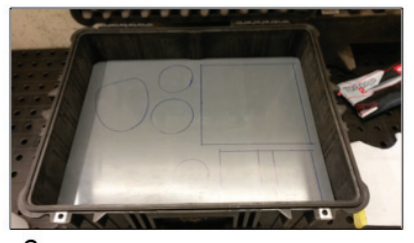

C

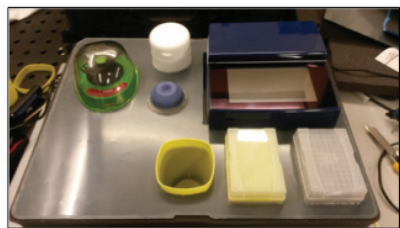

B

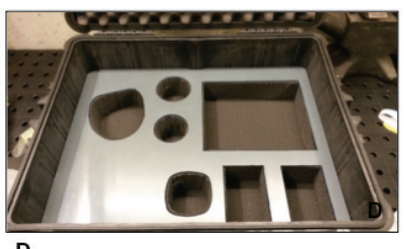

D

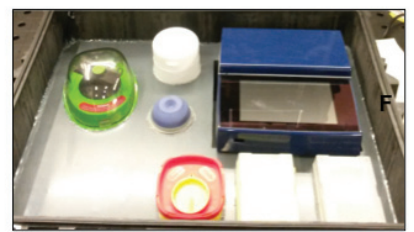

A: A PVC layer was placed on top of the foam filling the bottom of the suitcase.

B: Bespoke insert slots were cut out of the PVC and the foam layer to host the tubescanner, the box for the disinfection wipes, the waste container, the vortex, the minicentrifuge and two boxes of refill pipette tips.

C: Foam, PVC layer and instruments were assembled outside the suitcase. Electricity wires were stowed underneath the foam layer. The equipment was fixed to the PVC layer using hot glue.

D: The setup was placed into the suitcase and the seams were sealed with hot glue.

\section{Methods}

\section{Study design and samples}

The study was conducted during the 2014-15 EBOV outbreak in Guinea. On 23 March 2014, the Institute Pasteur de Dakar (IPD), Senegal, upon request of the WHO and the Guinean Ministry of Health deployed a mobile laboratory team to Conakry. An ETC was set up at Donka hospital in Conakry. Serum samples from acute cases and swabs (cheek and tongue) from deceased meeting the WHO definition of a suspected EVD case (see below) were collected in Conakry, Matoto, Télimélé, Coyah and other regions of Guinea between December 2014 and May 2015 and sent to our laboratory for diagnosis. In addition, following an upsurge of EVD cases connected to funeral rites, oral swabs from all deceased were tested at the morgue in Matoto in March and April 2015. During this study, the EBOV RT-RPA was evaluated in parallel to reference methods.

Suspected EVD cases were defined as any person, alive or dead, suffering or having suffered from a sudden onset of high fever and having had contact with a suspected, probable or confirmed case of EVD, or any person with sudden onset of high fever and at least three of the following symptoms: headaches, anorexia/ loss of appetite, lethargy, aching muscles or joints, 


\section{FIGURE 4}

Sensitivity of the Ebola RT-recombinase polymerase amplification test on (A) inactivated Ebola virus and (B) swab samples, Guinea, December 2014-May 2015 ( $\mathrm{n=138)}$

A.

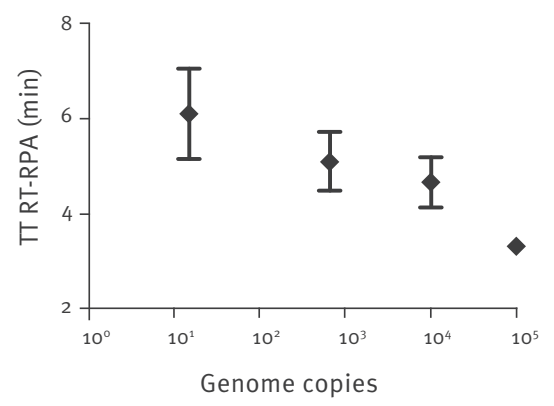

B.

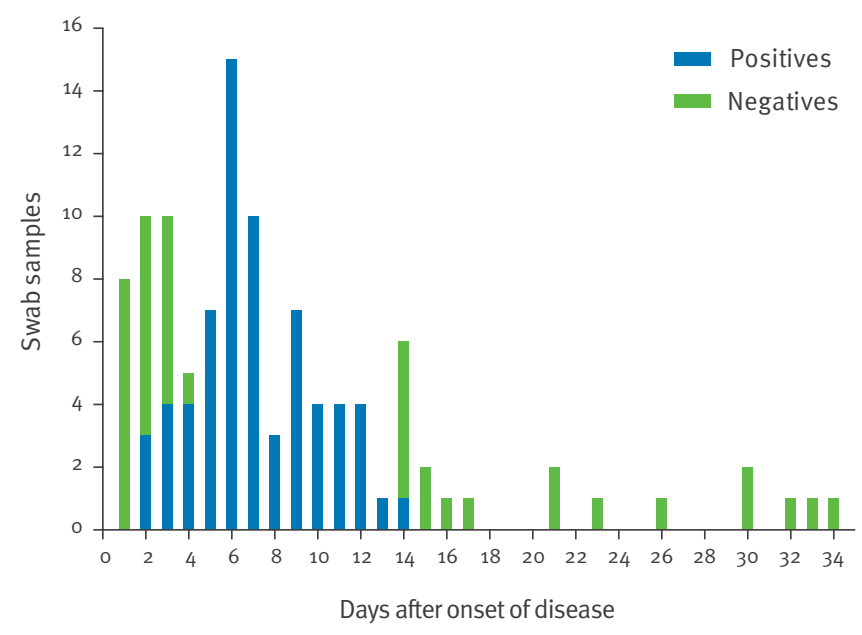

RPA: recombinase polymerase amplification; RT: reverse transcription; TT: threshold time.

A: Plasma samples spiked with inactivated Ebola virus (source: ENIVD) extracted and re-quantified by real-time PCR. Extracts $(1.5 \times 105,7.0 \times 102,1.0 \times 104,1.0 \times 105$ Ebola genome molecules /reaction) were tested in triplicate by RT-RPA. The detection limit was 15 Ebola genome molecules/reaction in a maximum of $8 \mathrm{~min}$.

B: Results from 138 swab samples from deceased suspected EVD cases with symptoms among a cohort of 928 samples from deceased with and without symptoms. Positive and negative results are scored over days after onset of disease.

breathing difficulties, and any person with inexplicable bleeding or any sudden inexplicable death.

\section{RNA extraction and inactivation}

Two extraction methods were used. In the first method, viral RNA was extracted from $100 \mu \mathrm{l}$ serum or swab transport medium using the QIAamp Viral Mini Kit (QC; Qiagen, Hilden, Germany). RNA was eluted in 50 $\mu \mathrm{l}$ Tris-EDTA buffer. The second extraction protocol (SpeedXtract Nucleic Acid Kit (SE), Qiagen, Hilden, Germany) was a reverse extraction method extracting protein debris by way of magnetic beads after an initial $10 \mathrm{~min}$ heating step at $95^{\circ} \mathrm{C}$. It yielded $200 \mu \mathrm{l}$ supernatant from $20 \mu \mathrm{l}$ of serum or oral swab transport fluid diluted 1:2 with molecular grade water.

We added $5 \mu \mathrm{l}$ of either eluate to the W-PCR (EBOV one-step real-time RT-PCR described in [6]) and the optimised RT-RPA, and $10 \mu \mathrm{l}$ to the A-PCR (The RealStar Filovirus RT-PCR Kit, Altona-Diagnostics, Hamburg, Germany).

To test for inactivation of EBOV by the new SE kit, SE extract dilutions from $10^{-1}$ to $10^{-5}$ were added in triplicate onto $2 \times 10^{5}$ VeroE6 cells in a 96-well plate and incubated for five days. The supernatant was passaged three times by transfer to a new well, followed by a $3 \mathrm{~h}$ incubation, a wash, and another $48 \mathrm{~h}$ incubation step. Finally, cells were washed three times and RNA was extracted in $200 \mu \mathrm{l}$ Trizol and submitted to an EBOV inhouse PCR. For each dilution, three more wells to which the supernatants had been added in the same manner were subjected to an immunofluorescence assay after passage 1 [8]. A not extracted patient serum sample was used as positive control and showed virus growth on VeroE6 cells.

\section{Real-time RT-PCR}

The W-PCR was performed on the SmartCycler (Cepheid, Sunnyvale, US) using the RNA Master Hybridisation Probes kit (Roche, Manheim, Germany). A dried 10-fold primer and probe mix containing 100 pmol EBOZ FP and EBOZ RP and 50 pmol EBOZ P (TIB Molbiol, Germany) was used. The A-PCR was used on the SmartCycler according to the manufacturer's instructions. Positive results above cycle threshold (Ct) 35 were regarded as equivocal and repeated for confirmation [9].

\section{RT-RPA assay}

The primers and the exo probe of an existing EBOV RT-RPA assay [7] were redesigned to adopt mismatches of the current EBOV outbreak strain (Figure 1, Table 1) following RPA design guidelines [10].

The RT-RPA was performed using a custom-made EBOVspecific exo RT kit with pellets containing optimised enzyme concentrations similar to the commercial TwistAmp RT exo kit $[10,11]$, and additionally containing primers and probe. Briefly, $5 \mu \mathrm{l}$ of RNA template and $45 \mu \mathrm{l}$ of customised rehydration buffer containing magnesium acetate were added to each pellet in a strip of eight tubes delivered in vacuum-sealed pouches. In each strip, tubes 1 to 5 were used to test samples, tube 6 was used as negative extraction control and tubes 7 and 8 for a negative and positive RT-RPA reaction control. The reaction tubes were mixed, centrifuged and then placed into the ESEQuant TS2 (QIAGEN Lake Constance $\mathrm{GmbH}$, Stockach, Germany) for real-time monitoring of fluorescence at $42{ }^{\circ} \mathrm{C}$ for $15 \mathrm{~min}$, with brief mixing and centrifugation of the reaction tubes after $4 \mathrm{~min}$. The resulting curves were analysed by TS2 Studio Version 1.8.2.0 (QIAGEN Lake Constance $\mathrm{GmbH}$, Stockach, Germany). Increase of fluorescence intensity over time above the mean background signal 
TABLE 1

Primers and probe designed for the updated Zaire ebolavirus RT-recombinase polymerase amplification assay

\begin{tabular}{|l|c|}
\hline Name & RPA primers and exo probes \\
\hline EBOG RPA FP & TGATCCRACTGACTCACAGGATACGACCATT*C \\
\hline EBOG RPA RP & TCTAGATCGAATAGGAYCAARTCATCTGGTGC*A \\
\hline EBOG RPAP & GATGATGGARGCTACGGCGATACCARAG-BTF-CTCGGAAACGGYATG-Ph \\
\hline
\end{tabular}

B: thymidine nucleotide-carrying blackhole quencher 1; F: thymidine nucleotide-carrying fluorescein; FP: forward primer; P: probe; Ph: 3’ phosphate to block elongation; RP: reverse primer; T: tetrahydrofuran spacer.

* phosphothioate backbone.

TABLE 2

Pathogen nucleic acids used for evaluation of the Ebola virus RT-recombinase polymerase amplification assay

\begin{tabular}{|c|c|c|c|c|}
\hline Pathogen & Strain/source & $\begin{array}{l}\text { Ebola RT-RPA } \\
\text { TT (min) }\end{array}$ & $\begin{array}{l}\text { Real-time } \\
\text { RT-PCR } \\
\text { CT value }\end{array}$ & $\begin{array}{l}\text { Real-time } \\
\text { RT-PCR assay }\end{array}$ \\
\hline Ebola virus & Zaire strain/BNI & $4 \cdot 7$ & 21.0 & \multirow{2}{*}{ ENIVD Ebola standard control and [6] } \\
\hline Ebola virus & GIN/2014/Gueckedou-C05/BNI & 5 & 25.4 & \\
\hline Sudan virus & Sudan Virus Maridi & Negative & 22.26 & {$[6]$} \\
\hline Bundibugyo virus & Bundibugyo virus & Negative & 28.7 & In-house assay \\
\hline Marburg virus & Musoke/BNI & Negative & 24.5 & [6] \\
\hline $\begin{array}{l}\text { Crimean Congo } \\
\text { haemorrhagic fever virus }\end{array}$ & $\begin{array}{l}\text { Kosova Hoti/BNI, } \\
\text { Afgog-299o/BNI }\end{array}$ & Negative & $\begin{array}{l}20.3 \\
22.4\end{array}$ & $\begin{array}{c}\text { RealStar CCHFV RT-PCR Kit ((Altona } \\
\text { Diagnostics) }\end{array}$ \\
\hline Lassa virus & $\begin{array}{c}\text { Josiah/BNI, } \\
\text { Lib } 1580 / 121 / \text { BNI }\end{array}$ & Negative & $\begin{array}{l}25.9, \\
34.7\end{array}$ & [16] \\
\hline Yellow fever virus & $\begin{array}{c}\text { Asibi AY640589.1 } \\
\text { 17D RKI }\end{array}$ & Negative & $\begin{array}{l}20.6 \\
20.0\end{array}$ & [17] \\
\hline Rift valley fever virus & Strain $\mathrm{ZH}_{548}$ & Negative & 26.2 & [18] \\
\hline Dengue virus $1-4$ & $\begin{array}{l}\text { VR344 (Thai } 1958 \text { strain), } \\
\text { VR345 (TH-36 strain), } \\
\text { VR216 (H87 strain), } \\
\text { VR217 (H241 strain) }\end{array}$ & Negative & $\begin{array}{l}24.2 \\
21.3 \\
23.1 \\
22.7 \\
\end{array}$ & In-house assay \\
\hline Zika virus & MR766 & Negative & 20.86 & [19] \\
\hline Chikungunya virus & A26 Strain & Negative & 25.13 & In-house assay \\
\hline Plasmodium falciparum & ND & Negative & 15.0 & In-house qualitative assay \\
\hline
\end{tabular}

BNI: Bernhard Nocht Institute; ND: not determined; GIN: Guinea; RKI: Robert Koch Institute; RPA: recombinase polymerase amplification; RT: reverse transcription; TT: threshold time.

Ebola RT-RPA assay identified only Zaire ebolavirus but not the nucleic acids of other pathogens.

was analysed by threshold validation (mV/min). Slope validation was used to verify that the increase of fluorescence occurred at a sufficiently high rate, and was verified by first derivative analysis.

\section{The mobile laboratory}

The mobile laboratory consisted of a glovebox (Bodo Koennecke, Berlin, Germany), a Diagnostics-in-aSuitcase (DiaS), and a solar panel and power pack set (Yeti 400 set, GOALZERO, South Bluffdale, US). The disassembled glovebox was kept in a metal box $(80 \times 60 \times 41 \mathrm{~cm})$ with other necessary materials (disinfectant solution, extraction kits, filter tips, racks, vortex, heat block, autoclavable plastic bags and personal protective equipment (PPE). The total weight was $28 \mathrm{~kg}$ for the box and $16 \mathrm{~kg}$ for the DiaS. Sample inactivation and RNA extraction using the SE kit were done in the glovebox (Figure $2 A, B$ ). This allowed handling of hazard group 4 samples. The RT-RPA assay was performed in the DiaS (Figure 2A,C) containing the ESEQuant TS2 device with integrated touchscreen to operate the device and display the results (Qiagen, Lake Constance $\mathrm{GmbH}$, Stockach, Germany). The DiaS was assembled using a trolley case $(63 \times 50 \times 30.2 \mathrm{~cm}$, Peli, Düsseldorf, Germany). The bottom layer of the DiaS contains foam to adsorb shocks during transportation which is covered by a PVC top layer fixed around inserted devices to provide water and chemical resistance (Figure 3, [12]).

\section{Statistical methods}

Data were analysed using $R$ (version 3.1.1) [13]. Performance parameters of the test (sensitivity (Se), specificity (Sp), positive (PPV) and negative predictive 
Evaluation of Ebola virus diagnostic assays, serum and swab samples, Guinea, December 2014-May 2015 (n=1,069)

\begin{tabular}{|c|c|c|c|c|c|c|c|c|c|c|c|c|c|}
\hline \multirow{2}{*}{$\begin{array}{l}\text { Extraction } \\
\text { method }^{\text {a }}\end{array}$} & \multirow{2}{*}{$\begin{array}{l}\text { Sample } \\
\text { type }\end{array}$} & \multirow{2}{*}{$\begin{array}{c}\text { Reference } \\
\text { test }\end{array}$} & \multirow{2}{*}{$\begin{array}{c}\text { Analysed } \\
\text { test }\end{array}$} & \multirow{2}{*}{ Ct range } & \multirow{2}{*}{$\begin{array}{l}\text { Analysis } \\
\text { values }^{b}\end{array}$} & \multirow{2}{*}{ PPV } & \multirow{2}{*}{ NPV } & \multirow{2}{*}{ Sensitivity } & \multirow{2}{*}{ Specificity } & \multirow{2}{*}{$\mathrm{n}$} & \multirow{2}{*}{$\begin{array}{l}\text { Analysed } \\
\text { method }\end{array}$} & \multicolumn{2}{|c|}{ Reference method } \\
\hline & & & & & & & & & & & & Positive & Negative \\
\hline \multirow{16}{*}{ QC } & \multirow{16}{*}{ Serum } & \multirow[b]{2}{*}{$A-P C R$} & \multirow[b]{2}{*}{ RT-RPA } & \multirow[b]{2}{*}{$0-40$} & \multirow{2}{*}{$\begin{array}{l}\text { Estimate: } \\
95 \% \mathrm{Cl}: \\
\mathrm{p} \text { value: }\end{array}$} & \multirow{2}{*}{$\begin{array}{c}0.82 \\
{[0.72-0.9]} \\
3.2 \times 10^{-9} \\
\end{array}$} & \multirow{2}{*}{$\begin{array}{c}0.97 \\
{[0.88-1.00]} \\
1.19 \times 10^{-14} \\
\end{array}$} & \multirow{2}{*}{$\begin{array}{c}0.97 \\
{[0.90-1.00]} \\
4.21 \times 10^{-18} \\
\end{array}$} & \multirow{2}{*}{$\begin{array}{c}0.79 \\
{[0.60-0.88]} \\
1.04 \times 10^{-6}\end{array}$} & \multirow[b]{2}{*}{141} & Positive & 68 & 15 \\
\hline & & & & & & & & & & & Negative & 2 & 56 \\
\hline & & \multirow[b]{2}{*}{$A-P C R$} & \multirow[b]{2}{*}{ RT-RPA } & \multirow[b]{2}{*}{130} & \multirow{2}{*}{$\begin{array}{l}\text { Estimate: } \\
95 \% \mathrm{Cl}: \\
\text { p value: }\end{array}$} & \multirow{2}{*}{$\begin{array}{c}0.46 \\
{[0.27-0.67]} \\
0.8450 \\
\end{array}$} & & 0.86 & 0.8 & & Positive & 12 & 14 \\
\hline & & & & & & & $\begin{array}{c}{[0.88-1.00]} \\
1.19 \times 10^{-14}\end{array}$ & $\begin{array}{c}{[0.57-0.98]} \\
0.0129\end{array}$ & $\begin{array}{c}{[0.69-0.89]} \\
4.30 \times 10^{-7}\end{array}$ & 84 & Negative & 2 & 56 \\
\hline & & & & & Estimate: & & 0.86 & 0.91 & & & Positive & 83 & o \\
\hline & & W-PCR & RT-RPA & $0-40$ & $\begin{array}{l}95 \% \mathrm{Cl}: \\
\text { p value: }\end{array}$ & $\begin{array}{c}{[0.93-1.00]} \\
0.178\end{array}$ & $\begin{array}{c}{[0.75-0.94]} \\
1.57 \times 10^{-8}\end{array}$ & $\begin{array}{l}{[0.83-0.96]} \\
7.58 \times 10^{-17}\end{array}$ & $\begin{array}{l}{[0.93-1.00]} \\
1.78 \times 10^{-15}\end{array}$ & 141 & Negative & 8 & 50 \\
\hline & & & & & Estimate: & 1 & 0.86 & 0.76 & 1 & & Positive & 26 & 0 \\
\hline & & W-PCR & RT-RPA & 130 & $\begin{array}{l}95 \% \mathrm{Cl}: \\
\text { p value: }\end{array}$ & $\begin{array}{l}{[0.87-1.00]} \\
2.98 \times 10^{-8} \\
\end{array}$ & $\begin{array}{c}{[0.75-0.94]} \\
1.57 \times 10^{-8}\end{array}$ & $\begin{array}{c}{[0.59-0.89]} \\
0.0029\end{array}$ & $\begin{array}{l}{[0.93-1.00]} \\
1.78 \times 10^{-15} \\
\end{array}$ & 84 & Negative & 8 & 50 \\
\hline & & & & & Estimate: & 0.77 & 1 & 1 & 0.7 & & Positive & 70 & 21 \\
\hline & & $A-P C R$ & W-PCR & $0-40$ & $\begin{array}{l}95 \% \mathrm{Cl}: \\
\text { p value: }\end{array}$ & $\begin{array}{c}{[0.67-0.85]} \\
2.51 \times 10^{-7} \\
\end{array}$ & $\begin{array}{l}{[0.93-1.00]} \\
1.78 \times 10^{-15} \\
\end{array}$ & $\begin{array}{l}{[0.95-1.00]} \\
1.69 \times 10^{-21} \\
\end{array}$ & $\begin{array}{c}{[0.58-0.81]} \\
0.0007\end{array}$ & 141 & Negative & 0 & 50 \\
\hline & & & & & Estimate: & 0.41 & 1 & 1 & 0.71 & & Positive & 14 & 20 \\
\hline & & $A-P C R$ & W-PCR & 130 & $\begin{array}{l}95 \% \mathrm{Cl}: \\
\text { p value: }\end{array}$ & $\begin{array}{c}{[0.25-0.59]} \\
0.392 \\
\end{array}$ & $\begin{array}{l}{[0.93-1.00]} \\
1.78 \times 10^{-15} \\
\end{array}$ & $\begin{array}{c}{[0.77-1.00]} \\
0.0001 \\
\end{array}$ & $\begin{array}{c}{[0.59-0.82]} \\
0.0004\end{array}$ & 84 & Negative & 0 & 50 \\
\hline & & & & & Estimate: & 1 & 0.7 & 0.77 & 1 & & Positive & 70 & 0 \\
\hline & & W-PCR & $A-P C R$ & $0-40$ & $\begin{array}{l}95 \% \mathrm{Cl}: \\
\text { p value: }\end{array}$ & $\begin{array}{l}{[0.95-1.00]} \\
1.69 \times 10^{-21}\end{array}$ & $\begin{array}{c}{[0.58-0.81]} \\
7.67 \times 10^{-4} \\
\end{array}$ & $\begin{array}{c}{[0.67-0.85]} \\
2.51 \times 10^{-7} \\
\end{array}$ & $\begin{array}{l}{[0.93-1.00]} \\
1.78 \times 10^{-15} \\
\end{array}$ & 141 & Negative & 21 & 50 \\
\hline & & & & & Estimate: & 1 & 0.71 & 0.41 & 1 & & Positive & 14 & 0 \\
\hline & & W-PCR & $A-P C R$ & 130 & $\begin{array}{l}95 \% \mathrm{Cl}: \\
\text { p value: }\end{array}$ & $\begin{array}{c}{[0.77-1.00]} \\
0.0001\end{array}$ & $\begin{array}{c}{[0.59-0.82]} \\
0.0004\end{array}$ & $\begin{array}{c}{[0.25-0.59]} \\
0.3920\end{array}$ & $\begin{array}{l}{[0.93-1.00]} \\
1.78 \times 10^{-15}\end{array}$ & 84 & Negative & 20 & 50 \\
\hline & & & & & Estimate: & 0.81 & 1 & 1 & 0.98 & & Positive & 67 & 16 \\
\hline & & $A-P C R$ & RT-RPA & $0-40$ & $\begin{array}{l}95 \% \mathrm{Cl}: \\
\text { p value: }\end{array}$ & $\begin{array}{c}{[0.71-0.89]} \\
1.39 \times 10^{-8} \\
\end{array}$ & $\begin{array}{l}{[1.00-1.00]} \\
1.20 \times 10^{-240}\end{array}$ & $\begin{array}{l}{[0.95-1.00]} \\
1.36 \times 10^{-20}\end{array}$ & $\begin{array}{l}{[0.97-0.99]} \\
2.86 \times 10^{-212}\end{array}$ & $881^{c}$ & Negative & 0 & 798 \\
\hline & & & & & Estimate: & 0.5 & 1 & 1 & 0.98 & & Positive & 15 & 15 \\
\hline SE & Swab & $A-P C R$ & RT-RPA & 130 & $\begin{array}{l}95 \% \mathrm{Cl}: \\
\text { p value: }\end{array}$ & $\begin{array}{c}{[0.31-0.69]} \\
1 \\
\end{array}$ & $\begin{array}{l}{[1.00-1.00]} \\
1.20 \times 10^{-240} \\
\end{array}$ & $\begin{array}{c}{[0.78-1.00]} \\
6.10 \times 10^{-5}\end{array}$ & $\begin{array}{l}{[0.97-0.99]} \\
1.12 \times 10^{-213}\end{array}$ & $828^{c}$ & Negative & 0 & 798 \\
\hline JL & Swato & & & & Estimate: & 1 & 1 & 1 & 1 & & Positive & 120 & o \\
\hline & & W-PCR & RT-RPA & $0-40$ & $\begin{array}{l}95 \% \mathrm{Cl}: \\
\text { p value: }\end{array}$ & $\begin{array}{l}{[0.96-1.00]} \\
4.14 \times 10^{-25} \\
\end{array}$ & $\begin{array}{l}{[0.99-1.00]} \\
3.69 \times 10^{-127}\end{array}$ & $\begin{array}{l}{[0.96-1.00]} \\
4.14 \times 10^{-25} \\
\end{array}$ & $\begin{array}{l}{[0.99-1.00]} \\
3.69 \times 10^{-127}\end{array}$ & 928 & Negative & 0 & 808 \\
\hline & & & & & Estimate: & 1 & 1 & 1 & 1 & & Positive & 55 & o \\
\hline & & W-PCR & RT-RPA & 130 & $\begin{array}{l}95 \% \mathrm{Cl}: \\
\text { p value: }\end{array}$ & $\begin{array}{c}{[0.88-1.00]} \\
3.73 \times 10^{-9}\end{array}$ & $\begin{array}{l}{[0.99-1.00]} \\
3.69 \times 10^{-127} \\
\end{array}$ & $\begin{array}{c}{[0.88-1.00]} \\
3.73 \times 10^{-9} \\
\end{array}$ & $\begin{array}{l}{[0.99-1.00]} \\
3.69 \times 10^{-127} \\
\end{array}$ & $863^{c}$ & Negative & 0 & 808 \\
\hline
\end{tabular}

$\mathrm{Cl}$ : confidence interval; CT: cycle threshold; NPV: negative predictive value; QC: QIAamp Viral Mini Kit; RPA: recombinase polymerase amplification; RT: reverse transcription; PPV: positive predictive value; SE: SpeedXtract nucleic acid extraction kit.

a Extraction method for RT-RPA. In all cases the reference test was tested with extracts from QC.

${ }^{b}$ Estimated proportions are given in decimals.

c This comparison was tested on a smaller subset.

values (NPV) were estimated for each of the assays using real-time RT-PCR assays as reference test. The $95 \%$ confidence interval (Cl) of performance parameters was calculated based on the exact binomial test. $P$ values are derived from the exact binomial test. The calculated Se and Sp were considered statistically significant for $p$ values $<0.05$. We used Fisher's exact test to compare RT-RPA performance parameters in comparison with W-PCR and A-PCR as the reference method at different $\mathrm{Ct}$ ranges.

\section{Results}

\section{Inactivation}

The inactivation of EBOV by the SE extraction procedure was confirmed in VeroE6 cells inoculated with SE extracts which were all negative in IFA. PCR results at passage 4 ranged from $\mathrm{Ct} 32$ to undetectable. Since the IFA was negative, the PCR results were assumed to be due to remnant input RNA but not to actively replicating virus.

\section{Analytical sensitivity and specificity of the RT- RPA assay}

W-PCR and RT-RPA detected RNA standards over a range of 5 to $5 \times 10^{5}$ genome copies (GC)/reaction and 50 to $5 \times 10^{5}$ genome copies/reaction, respectively. RT-RPA assays could detect as little as $5 \mathrm{GC} /$ reaction of a molecular RNA standard (data not shown) and 15 $\mathrm{GC} /$ reaction in EBOV-spiked human plasma samples (Figure $4 \mathrm{~A}$ ). No cross-detection of important differential diagnostic pathogens or any other filoviruses was observed for the Ebola RT-RPA assay (Table 2).

\section{Performance of RT-PCR and RT-RPA assay using sera}

Using a total of 141 sera extracted with QC, RT-RPA and $W-P C R$ performances were assessed using the WHO-approved A-PCR as reference. Against the 
TABLE 4

Significance of the performance analysis results for Ebola virus diagnostic assays, serum samples, Guinea, December 2014-May 2015 ( $\mathrm{n}=141)$

\begin{tabular}{|l|c|c|c|c|}
\hline$S e$ & All & 0.91 & 0.97 & 0.19 \\
\hline$S p$ & All & 1 & 0.79 & $3.45 \times 10^{-4}$ \\
\hline$P P V$ & All & 1 & 0.82 & $3.05 \times 10^{-5}$ \\
\hline$N P V$ & All & 0.86 & 0.97 & 0.09 \\
\hline$S e$ & $0-20$ & 1 & 1 & 1.00 \\
\hline$S p$ & $0-20$ & 1 & 1 & 1.00 \\
\hline$P P V$ & $0-20$ & 1 & 1 & 1.00 \\
\hline$N P V$ & $0-20$ & 1 & 1 & 1.00 \\
\hline$S e$ & $0-30$ & 1 & 1 & 1.00 \\
\hline$S p$ & $0-30$ & 1 & 0.98 & 1.00 \\
\hline$P P V$ & $0-30$ & 1 & 0.98 & 1.00 \\
\hline$N P V$ & $0-30$ & 1 & 1 & 1.00 \\
\hline$S e$ & $>30$ & 0.76 & 0.86 & 0.70 \\
\hline$S p$ & $>30$ & 1 & 0.80 & $3.27 \times 10^{-4}$ \\
\hline$P P V$ & $>30$ & 1 & 0.46 & $1.09 \times 10^{-5}$ \\
\hline$N P V$ & $>30$ & 0.86 & 0.97 & 0.09 \\
\hline
\end{tabular}

NPV: negative predictive value; RPA: recombinase polymerase amplification; RT: reverse transcription; PPV: positive predictive value; Se: Sensitivity; Sp: Specificity.

A-PCR, the RT-RPA yielded a lower PPV (82\% vs $100 \%$, $\left.p=3.05 \times 10^{-5}\right)$, a higher corresponding Se (97\% vs $91 \%, p=0.19)$, a higher NPV (97\% vs $86 \%, p=0.09)$ and a lower Sp $\left(79 \%\right.$ vs $\left.100 \%, p=3.45 \times 10^{-4}\right)$ than against the W-PCR (Table 3 rows 1 and 3, Table 4). The tendency of the results was even more pronounced in the subset of 84 samples with low viraemia (Ct values >30, Table 3 rows 2 and 4). The difference between the PCR assays was analysed and revealed a reduced Se (77\%) for the A-PCR compared with the W-PCR (Table 3 rows 7-8).

Samples determined as positive by the W-PCR but negative by the RT-RPA were also negative in the A-PCR, which missed some additional samples. There was no case of a negative RT-RPA result being positive in the A-PCR (Table 5).

\section{Performance of RT-PCR and RT-RPA assay using swabs}

In a preliminary test of RT-RPA efficiency on SE extracts from 47 swabs from deceased patients, all 47 samples scored positive in the W-PCR and the RT-RPA. Therefore, combined SE extraction and RT-RPA were deployed in the mobile laboratory and altogether 928 post-mortem swab samples (including the 47 preliminary ones) were tested. All 928 samples were also extracted by QC and tested by W-PCR and A-PCR. Overall, 120 samples scored positive both in W-PCR and RT-RPA, and only 67 of a subset of 83 samples scored positive in A-PCR. In reference to $\mathrm{QC}$ extraction and W-PCR, SE extraction

\section{TABLE 5}

Concordance of results from Ebola virus diagnostic assays, serum and swab samples, Guinea, December 2014-May $2015(n=928)$

\begin{tabular}{|l|c|c|c|}
\hline Sera & W-PCR & RT-RPA & A-PCR \\
\hline Positive & 91 & 83 & 70 \\
\hline Negative & 50 & 58 & 71 \\
\hline Total & 141 & 141 & 141 \\
\hline Swabs & W-PCR & RT-RPA & A-PCR \\
\hline Positive & 83 & 83 & 67 \\
\hline Negative & 798 & 798 & 814 \\
\hline Total & 881 & 881 & 881 \\
\hline
\end{tabular}

Forty-seven additional swab samples were only tested by W-PCR and RT-RPA. In $n=928$ samples, these two assays were absolutely concordant.

and RT-RPA yielded a Se and Sp of $100 \%$ each (PPV: $100 \%$; NPV: $100 \%)$. Since the results of W-PCR and RT-RPA were concordant, the significance of the results was not calculated (Table 5).

The prevalence of positives as tested by W-PCR and RT-RPA in the 928 swabs was $12.9 \%$. Of the 928 postmortem samples tested, 790 were from suspected cases for whom no signs of disease were recorded and 138 from suspected cases for whom information on symptoms and onset of disease ranging from 1 to 35 days before death were available. Of the 120 positive cases, 53 belonged to the group without recorded symptoms and 67 belonged to the group with symptoms. Positive results were most frequent around day 6 after disease onset and no positive results were obtained later than 14 days after onset of disease (Figure 4B).

\section{Deployment of the mobile laboratory to the local hospital in Guinea}

The mobile laboratory was easy to transport to the point of need (Figure 2D-F). The setup of the mobile laboratory including the assembly of the glovebox and donning the PPE took ca $30 \mathrm{~min}$. The SE step was performed in the glovebox for up to 10 samples in $30 \mathrm{~min}$, while the RT-RPA needed 20 min including pipetting steps and mixing. We were able to power the mobile laboratory (peak energy need: $173 \mathrm{~W}$ ) with the solar battery for up to 16 hours. Before moving to another spot, the glovebox and DiaS were disinfected with $2 \%$ bleach or $0.5 \%$ incidine. Altogether, setup, operation and disassembly of the unit was easy to perform in a timely manner.

Four Guinean biologists were equipped with and trained in the use of the mobile laboratory at the IPD in January 2015 in a five-day course. After a pilot phase in Guinea, the mobile laboratories were deployed in the Matoto district of Conakry to support testing of swabs from dead suspected cases, which was introduced to 
improve community engagement in the EBOV response as well as community surveillance.

\section{Discussion}

In this study, we evaluated the analytical and clinical performance of an updated EBOV RT-RPA compared with reference real-time RT-PCR assays. The isothermal RT-RPA assay, which allows real-time detection of amplification from RNA samples using primers and a fluorescent restriction probe within 3 to $15 \mathrm{~min}$ [10]. We improved this assay by adaptating the primers to the new sequences of the EBOV strain circulating in West Africa and incorporating them into dried RT-RPA pellets.

In sera extracted by QC, the RT-RPA scored a Se of $91 \%$ and $\mathrm{Sp}$ of $100 \%$ in reference to the W-PCR (Se: $97 \%$ and Sp: $79 \%$ in reference to the A-PCR), which means it would miss out some weak positives while identifying all true negatives correctly. Results from SE extracted sera were similar (data not shown). Taking swabs is less invasive than taking serum, which makes it more acceptable to populations, but is also safer and easier for sampling and testing. Since SE extraction does not require the use of a centrifuge, we tried to combine the RT-RPA with SE extraction of swabs to simplify our mobile laboratory procedure.

During the analysis of the results, we noted that the widely used A-PCR was less sensitive than the W-PCR. This lower Se was also described by other teams in Guinea and Sierra Leone $[14,15]$. A rapid detection test (ReEBOAg, Corgenix, Denver, US) was recently scored against the A-PCR with a Se of $91.8 \%$ and Sp of $84.6 \%$ and approved by the WHO for emergency use. Another recently described rapid detection test also scored a Se of $100 \%$ and a Sp of $96.6 \%$ against the A-PCR and was rated as a rule-out screening test by the authors because it would include all positives but would miss out on excluding all true negatives, therefore requiring a confirmatory test [15]. Our data confirm their interpretation that the performance of these tests was underestimated when using the A-PCR as reference test.

Our data show that the combination of SE and RT-RPA is superior to the above rule-out tests as all true positive and negative post-mortem oral swabs are detected. Our previous work has shown that magnetic bead extraction is preferable to centrifuge-based extraction under field conditions as it obviates the need for a high-speed centrifuge (unpublished data). We therefore tested the novel magnetic bead-based SE extraction with its 15 min protocol. The materials for both SE extraction and RT-RPA are stable at ambient temperature $\left(30-35^{\circ} \mathrm{C}\right)$ for up to three months and this cold chain-independent combination proved to be well suited for field diagnostics. It scored very satisfactory results in swab extracts (Table 3, rows 13-14), indicating that the RT-RPA does not need a confirmatory test and can be used on site to correctly include positives and exclude negatives.
The prevalence of EBOV in the 928 swabs tested was $12.9 \%$. The day of death after onset of disease peaked at day 6 (range: 2-14 days) in the group of 67 swabpositive deceased for whom disease symptoms were recorded. For the ongoing EBOV outbreak in West Africa, the mean day of symptom onset is 11 days after infection and sera should ideally be collected during the acute phase of illness, within the first 10 days of the disease [2]. We show here that the same is true for swabs, which could simplify diagnostics tremendously. In 53 positive cases, symptoms were not recorded, which was mainly due to a lack of information in the records of the Safe and Dignified Burial teams that did the sampling.

When new EVD foci erupted in previously not affected western parts of Conakry in April 2015, the mobile laboratory was deployed to Matoto to support teams in charge of safe and dignified burials. Since it had been decided that all deceased should be tested, these teams collected swab samples from deceased of five neighbourhoods of Conakry (Matoto, Ratoma, Dixinn, Matam and Kaloum) and up to 50 samples had to be tested per day. The emergency response results were provided every 30 to $60 \mathrm{~min}$ to the field investigators and physicians. The rapidity and mobility of the RT-RPA method in the DiaS, in comparison with the average 3 to $4 \mathrm{~h}$ turnover with regular real-time RT-PCR, was appreciated by burial teams, health authorities, response teams and communities, as it allowed rapid clearance for normal burials deceased persons who were confirmed negative. The results also encourage the use of swabs from patients at ETCs. In that context, it would still be necessary to determine if swab samples can replace sera samples.

The deployment demonstrated that the mobile laboratory using glovebox, DiaS, SE and RT-RPA is a very good solution for decentralised biosafe diagnosis of EBOV, resulting in direct impact on community engagement for disease control. Moreover, this small mobile laboratory run by local teams is a sustainable contribution to future outbreak control.

\section{Addendum (28 January 2016)}

The World Health Organisation (WHO)-approved RealStar Filovirus Screen RT-PCR Kit 1.0 (Altona Diagnostics, Hamburg, Germany) has been widely used on Smart Cyclers throughout the Ebola outbreak in West Africa, although the manufacturer does not list this device in their instructions for use. The company published a note on its website on 10 July 2015, commenting the reported lack of sensitivity of this kit when used on a Smart Cycler. According to our findings, we consider that the reported lack of sensitivity is related to the restriction of using the kit for specific PCR cyclers only, as also found by other groups [20] previously.

\section{Acknowledgements}

This study was funded by the Welcome Trust programme: Research for Health in Humanitarian Crises (R2HC) 13376: Point-of-care diagnostic testing for Ebola virus disease in 
Ebola treatment centres. We would like to thank Christiane Stahl-Hennig for her support and are indebted to Marvin Kulp and Thorsten Töteberg of the technical maintenance department of the German Primate Center who assisted in the assembly of the mobile suitcase laboratory. We also thank Merle Hanke from Qiagen Lake Constance and Andy Wende form Qiagen for technical advice on the TS2 and the SE kit. The inactivated virus preparation was kindly provided by Bernhard-Nocht-Institute, Hamburg, Germany. The sample preparation was performed at the Robert Koch-Institute in Berlin, Germany under the grant from the European Centre for Disease Prevention and Control (ECDC) and was provided by the European Network for Diagnostics of "Imported" Viral Diseases (ENIVD). We would like to thank the local WHO team in Guinea for assisting with material and fast track shipment procedures. We thank Gunnel Lindgren for expert technical assistance. We thank all partners involved in outbreak response teams especially WHO, Médecins Sans Frontières, International and Guinean Red Cross, Regulation team of Matoto district. We would also like to acknowledge Ibrahima Khalil Baldé, Amadou Doré, Hadja Aissatou Bah, Fodé Kourouma, Jacob Camara, Joseph Akoi for their excellent technical expertise during the deployment the mobile laboratories.

\section{Conflict of interest}

Oliver Nentwich and Olaf Piepenburg are employees of TwistDx Ltd, a wholly owned subsidiary of Alere Inc. The RPA technology is subject to background IP protection and is owned by Alere.

\section{Authors' contributions}

AAS, MW, AAEW, PP, OuF designed the study. AAS, MW, AAEW, PP wrote the manuscript. OuF, OsF, BS, AM, DK, AAS, NM collected the data. ON, OP developed and provided primer-in pellets, GF, SK, NF, MKK, AAD, LK, MN organize and support the field deployment. HK, AM performed the inactivation study. All authors contributed to analyse the data and reviewed the manuscript.

\section{Reference}

1. ColebundersR, TshombaA, Van KerkhoveMD, BauschDG, CampbellP, LibandeM, et al. Marburg hemorrhagic fever in Durba and Watsa, Democratic Republic of the Congo: clinical documentation, features of illness, and treatment. J Infect Dis. 2007;196(s2) Suppl 2;S148-53. DOI: 10.1086/520543 PMID: 17940943

2. WHO Ebola Response Team, Ebola virus disease in West Africa--the first 9 months of the epidemic and forward projections.N Engl J Med. 2014;371(16):1481-95. DOI: 10.1056/ NEJMoa1411100 PMID: 25244186

3. Reusken C, Niedrig M, Pas S, Anda P, Baize S, Charrel R, et al. Identification of essential outstanding questions for an adequate European laboratory response to Ebolavirus Zaire West Africa 2014. J Clin Virol. 2015;62:124-34.

4. World Health Organization (WHO). Ebola situation report 13 May 2015. Geneva: WHO; 2015. Available from: http://apps.who.int/iris/bitstream/10665/170508/1/ roadmapsitrep_13May15_eng.pdf?ua=

5. KranasterR, DrumM, EngelN, WeidmannM, HufertFT, MarxA. One-step RNA pathogen detection with reverse transcriptase activity of a mutated thermostable Thermus aquaticus DNA polymerase.Biotechnol J. 2010;5(2):224-31. DOI: 10.1002/ biot.200900200 PMID: 20108275

6. WeidmannM, MühlbergerE, HufertFT. Rapid detection protocol for filoviruses.J Clin Virol. 2004;30(1):94-9. DOI: 10.1016/j. jcv.2003.09.004 PMID: 15072761

7. EulerM, WangY, HeidenreichD, PatelP, StrohmeierO, HakenbergS, et al. Development of a panel of recombinase polymerase amplification assays for detection of biothreat agents. J Clin Microbiol. 2013;51(4):1110-7. DOI: 10.1128/ JCM.02704-12 PMID: 23345286
8. SalataC, BaritussioA, MunegatoD, CalistriA, HaHR, BiglerL, et al. Amiodarone and metabolite MDEA inhibit Ebola virus infection by interfering with the viral entry process. Pathog Dis. 2015;73(5):ftv032. DOI: 10.1093/femspd/ftv032 PMID: 25933611

9. Gunson RN, Collins TC, Carman WF. Practical experience of high throughput real time PCR in the routine diagnostic virology setting. J Clin Virol. 2006;35(4):355-67.

10. Piepenburg0, WilliamsCH, StempleDL, ArmesNA. DNA detection using recombination proteins.PLoS Biol. 2006;4(7):e204. DOI: 10.1371/journal.pbio.0040204 PMID: 16756388

11. Euler M, Wang Y, Nentwich O, Piepenburg O, Hufert FT, Weidmann M. Recombinase polymerase amplification assay for rapid detection of Rift Valley fever virus. J Clin Virol. 2012;54(4):308-12.

12. Abd El WahedA, WeidmannM, HufertFT. Diagnostics-in-aSuitcase: Development of a portable and rapid assay for the detection of the emerging avian influenza $A\left(\mathrm{H}_{7} \mathrm{~N} 9\right)$ virus.J Clin Virol. 2015;69:16-21. DOI: 10.1016/j.jcv.2015.05.004 PMID: 26209370

13. Team RDCR. A language and environment for statistical computing. Computing RFfS, editor. Vienna, Austria 2008.

14. JanvierF, GorbatchS, QuevalL, TopJ, VigierC, CotteJ, et al. Difficulties of interpretation of Zaire Ebola Virus PCR results and implication in the field. J Clin Virol. 2015;67:36-7. DOI: 10.1016/j.jcv.2015.04.001 PMID: 25959155

15. Walker NF, Brown CS, Youkee D, Baker P, Williams N, Kalawa A, et al. Evaluation of a point-of-care blood test for identification of Ebola virus disease at Ebola holding units, Western Area, Sierra Leone, January to February 2015. Euro Surveill. 2015;20(12):21073.

16. GireSK, GobaA, AndersenKG, SealfonRS, ParkDJ, KannehL, et al. Genomic surveillance elucidates Ebola virus origin and transmission during the 2014 outbreak. Science. 2014;345(6202):1369-72. DOI: 10.1126/science.1259657 PMID: 25214632

17. WeidmannM, FayeO, FayeO, KranasterR, MarxA, NunesMR, et al. Improved LNA probe-based assay for the detection of African and South American yellow fever virus strains. J Clin Virol. 2010;48(3):187-92. DOI: 10.1016/j.jcv.2010.04.013 PMID: 20556888

18. WeidmannM, Sanchez-SecoMP, SallAA, LyPO, ThionganeY, LôMM, et al. Rapid detection of important human pathogenic Phleboviruses. J Clin Virol. 2008;41(2):138-42. DOI: 10.1016/j. jcv.2007.10.001 PMID: 18006376

19. Faye O, FayeO, DialloD, DialloM, WeidmannM, SallAA. Quantitative real-time PCR detection of Zika virus and evaluation with field-caught mosquitoes.Virol J. 2013;10(1):311. DOI: $10.1186 / 1743-422$ X-10-311 PMID: 24148652

20. Janvier F, Sagui E, Foissaud V. ReEBOV Antigen Rapid Test kit for Ebola.Lancet. 2015;386(10010):2254-5. DOI: 10.1016/ So140-6736(15)01107-1 